\title{
Non-Fourier Heat Conduction Analysis with Temperature-Dependent Thermal Conductivity
}

\author{
H. Rahideh, ${ }^{1}$ P. Malekzadeh, ${ }^{2}$ and M. R. Golbahar Haghighi ${ }^{2}$ \\ ${ }^{1}$ Department of Chemical Engineering, Persian Gulf University, Bushehr 75168, Iran \\ ${ }^{2}$ Department of Mechanical Engineering, Persian Gulf University, Bushehr 75168, Iran
}

Correspondence should be addressed to P. Malekzadeh, p_malekz@yahoo.com

Received 27 January 2011; Accepted 10 March 2011

Academic Editor: M. Salvia

Copyright () $2011 \mathrm{H}$. Rahideh et al. This is an open access article distributed under the Creative Commons Attribution License, which permits unrestricted use, distribution, and reproduction in any medium, provided the original work is properly cited.

\begin{abstract}
As a first endeavor, the one- and two-dimensional heat wave propagation in a medium subjected to different boundary conditions and with temperature-dependent thermal conductivity is studied. Both the spatial as well as the temporal domain is discretized using the differential quadrature method (DQM). This results in superior accuracy with fewer degrees of freedom than conventional finite element method (FEM). To verify this advantage through some comparison studies, a finite element solution ise also obtained. After demonstrating the convergence and accuracy of the method, the effects of different parameters on the temperature distribution of the medium are studied.
\end{abstract}

\section{Introduction}

Most of heat conduction or mass diffusion problems are described and analyzed using Fourier's law and Fick's law. It is well known that these classical diffusion theories may break down when one is interested in the transient problems in an extremely short period of time, very high flux, or for very low temperatures. Then, a modified flux model for the transfer processes with a finite speed wave is suggested. The hyperbolic heat conduction equation based on the Cattaneo model for the heat flux incorporates a relaxation mechanism in order to gradually adjust to a change in the temperature gradient. This model has been a satisfactory extension of classical diffusion theory and can yield the hyperbolic diffusion equation within the continuum assumption.

During the past few years, considerable attention has been concerned with using the non-Fourier heat conduction in problems with very low temperatures, extremely short period of time, or very high heat flux; see, for example, [1-5].

There are some interesting analytical approaches for different non-Fourier heat conduction problems [6-11]. However, due to difficulty in applying analytical schemes to investigate problems with complicated geometries, boundary conditions, or variable thermal properties, attention to numerical schemes has been increased.
Carey and Tsai [12] applied central and backward difference schemes to a one-dimensional problem to examine oscillations for the numerical solution of propagating heat wave reflected at a boundary. Glass et al. [13] used McCormack's predictor-corrector method to solve the same problem. Tamma and Railkar [14] used finite element method with special shape functions to remove the numerical oscillations. Han-Taw and Jae-Yuh [15] solved one-dimensional hyperbolic heat equation using Laplacetransform and finite volume technique. They extended the presented approach for two-dimensional problems [16]. Yeung and Lam [17] developed a flux-splitting algorithm based on the Godunov numerical scheme for the solution of the one- and two-dimensional non-Fourier heat conduction equation. Hsu and Chu [18] used the central finite difference method to investigate the two- and threedimensional inverse non-Fourier heat conduction problem in electronic device. Liu and Chen [19] applied a hybrid application of the Laplace transform and control-volume formulation in-conjunction with the hyperbolic shape functions to investigate the hyperbolic diffusion problems. Wu and $\mathrm{Li}[20]$ extended the time discontinuous Galerkin finite element method (DGFEM) developed by Li et al. [21] to the heat wave propagation problem. Wu and Li [22] employed a Galerkin finite element method to simulate the heat wave 
propagation in the medium subjected to different kinds of heat source. Chen [23] combined the Laplace transform, the weighting function scheme, and the hyperbolic shape functions to investigate the effect of the surface curvature of a solid body on hyperbolic heat conduction. Reverberi et al. [24] proposed a numerical study on a nonlinear hyperbolic diffusion equation. The Hartree hybrid method, combining the finite difference techniques with the method of characteristics, was used in the presence of discontinuities between initial and boundary conditions. A sequential method was proposed to estimate boundary condition of the two-dimensional hyperbolic heat conduction problems by Yang [25]. An inverse solution was deduced from a finite difference method, the concept of the future time and a modified Newton-Raphson method. The numerical solution of Dorao [26] was based on the time-space least-squares spectral method. A spectral element method was applied for solving the hyperbolic system treating the heat flux as an independent variable in addition to temperature. T. M. Chen and C. C. Chen $[27,28]$ investigated the hyperbolic heat conduction problems in the radial-spherical coordinate system by the hybrid Green's function method. Wang [29] used the finite element method with linear least-squares error method to construct a simple noniterative inverse operation procedure for solving non-Fourier heat transfer effect in irregular geometry. The method combines the Laplace transform for the time domain, Green's function for the space domain, and $\varepsilon$-algorithm acceleration method for fast convergence of the series solution. Andarwa and Basirat Tabrizi [30] studied heat and mass transfer coupling under non-Fourier effect. Hsu [31] employed the conventional differential quadrature method to solve the hyperbolic heat conduction problem with constant material properties.

In this work, as a first endeavor, the incremental differential quadrature method (IDQM) is employed for the nonlinear non-Fourier one- and two-dimensional heat-transfer analysis. The initial conditions are directly implemented into the governing differential equations through the IDQM discretization rules without reformulating the IDQM weighting coefficients. The Newton-Raphson algorithm is employed to solve the nonlinear system of algebraic equations. After demonstrating the convergence and accuracy of the method, some parameter studies are performed.

\section{Basic Formulations and Solution Procedure}

Consider a rectangular domain with arbitrary thermal conditions along its boundaries. Its thermal conductivity $(k)$ is assumed to be a linear function of temperature [32]

$$
k(T)=k_{0}\left[1+\lambda\left(T-T_{0}\right)\right] .
$$

The other material properties of the domain such as density $(\rho)$ and specific heat capacity $\left(C_{p}\right)$ are assumed to be constant.

The hyperbolic constitutive equation governing the transient heat transfer may be written as

$$
\tau \frac{\partial q}{\partial t}+q=-k \nabla T
$$

where $\tau$ is the relaxation time, which is related to the thermal wave speed and thermal diffusivity as $\tau=\alpha / C^{2}$. Moreover, the energy equation can be written as

$$
\rho C_{p} \frac{\partial T}{\partial t}+\nabla \cdot q=g(x, y, t)
$$

Using (1) and (2), (3) takes the following form:

$$
\begin{gathered}
\left(\frac{\partial k}{\partial x} \frac{\partial T}{\partial x}+\frac{\partial k}{\partial y} \frac{\partial T}{\partial y}\right)+k\left(\frac{\partial^{2} T}{\partial x^{2}}+\frac{\partial^{2} T}{\partial y^{2}}\right)+g(x, y, t)+\tau \frac{\partial g}{\partial t} \\
=\tau \rho C_{p} \frac{\partial^{2} T}{\partial t^{2}}+\rho C_{p} \frac{\partial T}{\partial t}
\end{gathered}
$$

Due to nonlinear terms in (4), if it is not impossible to solve it analytically, it is very difficult to obtain such a solution. Hence, the approximate method should be used to solve it. As a first attempt, the differential quadrature method as an efficient and accurate numerical tool [31-36] is employed to solve (4) under arbitrary boundary and initial conditions.

The differential quadrature method (DQM) is an alternative discretization approach for directly solving the governing equations in mathematics and engineering applications. Here, the DQM is used to discretize both the spatial and temporal domains. According to DQ method, the domain is discretized into a set of $N_{x}$ and $N_{y}$ discrete grid points in the $x$ - and $y$-direction, respectively. Then, at a given grid point $\left(x_{i}, y_{j}\right)$, the first- and second-order derivatives of an arbitrary function $f(x, y, t)$ can be approximated as

$$
\begin{aligned}
\left.\frac{\partial f(x, y, t)}{\partial x}\right|_{\left(x_{i}, y_{j}\right)}=\sum_{m=1}^{N_{x}} A_{i m}^{x} f\left(x_{m}, y_{j}, t\right)=\sum_{m=1}^{N_{x}} A_{i m}^{x} f_{m j}(t) \\
\left.\frac{\partial^{2} f(x, y, t)}{\partial x^{2}}\right|_{\left(x_{i}, y_{j}\right)}=\sum_{m=1}^{N_{x}} B_{i m}^{x} f_{m j}(t) \\
\left.\frac{\partial f(x, y, t)}{\partial y}\right|_{\left(x_{i}, y_{j}\right)}=\sum_{n=1}^{N_{y}} A_{j n}^{y} f_{i n}(t) \\
\left.\frac{\partial^{2} f(x, y, t)}{\partial y^{2}}\right|_{\left(x_{i}, y_{j}\right)}=\sum_{n=1}^{N_{y}} B_{j n}^{y} f_{i n}(t)
\end{aligned}
$$

where $i=1,2, \ldots, N_{x}$ and $j=1,2, \ldots, N_{y}$.

In order to determine the weighting coefficients, a set of test functions should be used in (5). For polynomial basis functions DQ, a set of Lagrange polynomials is employed as the test functions. The normalized weighting coefficients for 
the first- and second-order derivatives in the $\zeta$-direction $(\zeta=$ $x, y$, and $t$ ) are thus determined, respectively, as

$$
\begin{aligned}
& A_{i j}^{\zeta}=\left\{\begin{array}{ll}
\frac{1}{L_{\zeta}} \frac{M\left(\zeta_{i}\right)}{\left(\zeta_{i}-\zeta_{j}\right) M\left(\zeta_{j}\right)} & i \neq j, \\
-\sum_{k=1, k \neq i}^{N_{\zeta}} A_{i k}^{\zeta} & i=j,
\end{array}, i, j=1,2, \ldots, N_{\zeta},\right. \\
& B_{i j}^{\zeta}=\left\{\begin{array}{lc}
2\left[A_{i i}^{\zeta} A_{i j}^{\zeta}-\frac{A_{i j}^{\zeta}}{\zeta_{i}-\zeta_{j}}\right] & i \neq j, \\
-\sum_{k=1, k \neq i}^{N_{\zeta}} B_{i k}^{\zeta} & i=j, j=1,2, \ldots, N_{\zeta},
\end{array}\right.
\end{aligned}
$$

where,

$$
M\left(\zeta_{i}\right)=\prod_{j=1, i \neq j}^{N_{\zeta}}\left(\zeta_{i}-\zeta_{j}\right)
$$

In numerical calculation, Chebyshev-Gauss-Lobatto quadrature points are used to generate the grid points, that is,

$$
\zeta_{i}=\frac{L_{\zeta}}{2}\left\{1-\cos \left[\frac{(i-1) \pi}{N_{\zeta}-1}\right]\right\} \quad i=1,2, \ldots, N_{\zeta}, \zeta=x, y, t .
$$

Also, to accurately and computationally efficient discretization of the temporal domain, the incremental DQ method (IDQM) is employed [32, 33, 36]. Based on this approach, the total temporal domain is divided into a set of subdomain and in each subdomain, the DQ rule is employed to discretize the temporal derivatives. At the end of each subdomain, the temperature and its first-time derivative $(\partial T / \partial t)$ are used as the initial conditions for the next subdomain.

In order to overcome the drawback of the conventional DQM for initial value problem with derivative of order greater or equal to two, which results in multiple initial conditions at the beginning of each temporal subdomain, the proposed method by Malekzadeh et al. [36] is employed. According to this methodology, the initial conditions are directly implemented through the time derivative at the temporal domain grid points $k\left(=2,3, \ldots, N_{T}\right)$ in the governing equation as

$$
\begin{aligned}
\left.\frac{d T_{i j}}{d t}\right|_{t=t_{k}}= & \sum_{m=2}^{N_{t}} A_{k m}^{t} T_{i j m}+A_{k 1}^{t} T_{i j 1}, \\
\left.\frac{d^{2} T_{i j}}{d t^{2}}\right|_{t=t_{k}}= & \sum_{n=2}^{N_{t}} \sum_{m=2}^{N_{t}} A_{k m}^{t} A_{m n}^{t} T_{i j n}, \\
& +\left.A_{k 1}^{t} \frac{d T_{i j}}{d t}\right|_{t=t_{1}}+\left(\sum_{m=2}^{N_{t}} A_{k m}^{t} A_{m 1}^{t}\right) T_{i j 1},
\end{aligned}
$$

where $T_{i j 1}$ and $d T_{i j} /\left.d t\right|_{t=t_{1}}$ are the initial values of the temperature and its first-order derivative at the beginning of an arbitrary temporal subdomain.
Based on the DQ discretization rules, the DQ discretized form of the governing (4) can be written as

$$
\begin{aligned}
& \left(\frac{\partial k}{\partial x}\right)_{i j k} \sum_{m=1}^{N_{x}} A_{i m}^{x} T_{m j k}+\left(\frac{\partial k}{\partial y}\right)_{i j k} \sum_{n=1}^{N_{y}} A_{j n}^{y} T_{i n k} \\
& \quad+k_{i j k}\left(\sum_{m=1}^{N_{x}} B_{i m}^{x} T_{m j k}+\sum_{n=1}^{N_{y}} B_{j n}^{y} T_{i n k}\right)+g_{i j k}+\tau\left(\frac{\partial g}{\partial t}\right)_{i j k} \\
& =\tau \rho C_{p}\left[\sum_{n=2}^{N_{t}} \sum_{m=2}^{N_{t}} A_{k m}^{t} A_{m n}^{t} T_{i j n}+\left.A_{k 1}^{t} \frac{d T_{i j}}{d t}\right|_{t=t_{1}}\right. \\
& \left.+\left(\sum_{n=2}^{N_{t}} A_{k n}^{t} A_{n 1}^{t}\right) T_{i j 1}\right] \\
& +\rho C_{p}\left(\sum_{m=2}^{N_{t}} A_{k m}^{t} T_{i j m}+A_{k 1}^{t} T_{i j 1}\right) .
\end{aligned}
$$

In a similar manner, the boundary conditions can be discretized. An iterative algorithm based on the NewtonRaphson method is employed to solve the resulting nonlinear algebraic system of equations in each temporal subdomain to obtain the temperature distribution at the spatial grid points and at each time step.

\section{Numerical Results}

3.1. One-Dimensional Problems. In order to demonstrate the accuracy of the method, a non-Fourier one-dimensional transient heat conduction problem subjected to two different types of initial conditions for which the exact solution was obtained by Wang [6] is considered. The boundary and initial conditions are taken as follows.

Boundary conditions:

$$
T(0, t)=0, \quad T\left(L_{x}, t\right)=0,
$$

Initial conditions:

$$
\left\{\begin{array}{l}
\text { Type }(1): T(x, 0)=0, \quad \frac{\partial T(x, 0)}{\partial t}=1, \\
\text { Type }(2): T(x, 0)=\sin \left(\frac{\pi x}{L_{x}}\right), \quad \frac{\partial T(x, 0)}{\partial t}=0 .
\end{array}\right.
$$

Also, in preparation of the numerical results, the values of the relaxation time and thermal diffusivity are assumed to be unit ( $\tau=1, \alpha=1)$. The results for the time history of the temperature at the center of the domain $(\xi=0.5)$ and also the temperature distribution along the domain at the nondimensional time $t^{*}=0.5$ are presented in Figures 1 and 2, respectively. Excellent agreement between the results of the presented IDQM and the exact solution [6] is obvious.

As another example, a comparison study between the results of the presented method and the finite element method (FEM) for a problem with specified, time-varying 


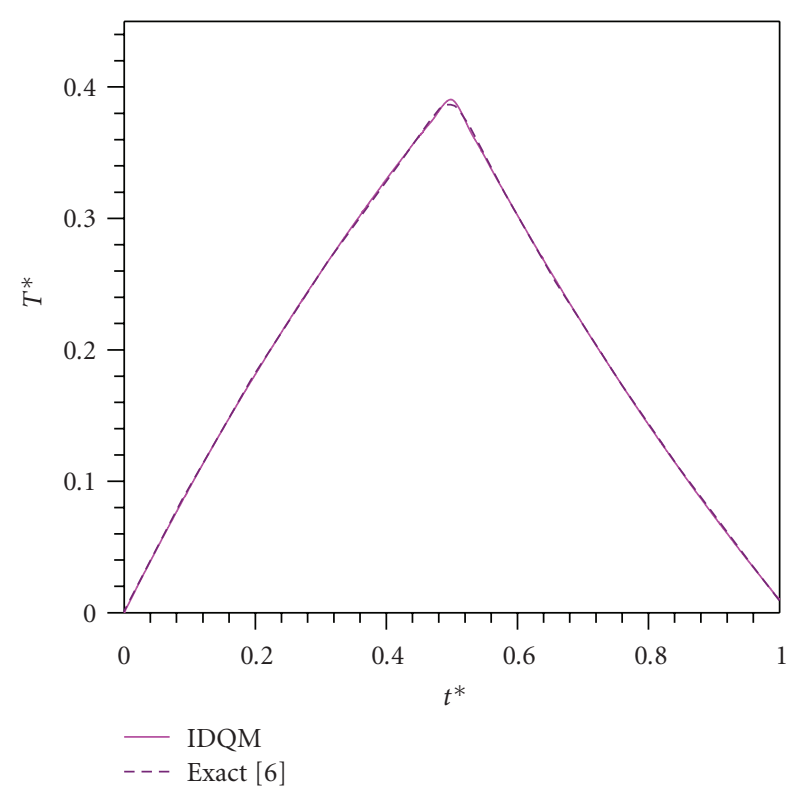

(a) Type (1)

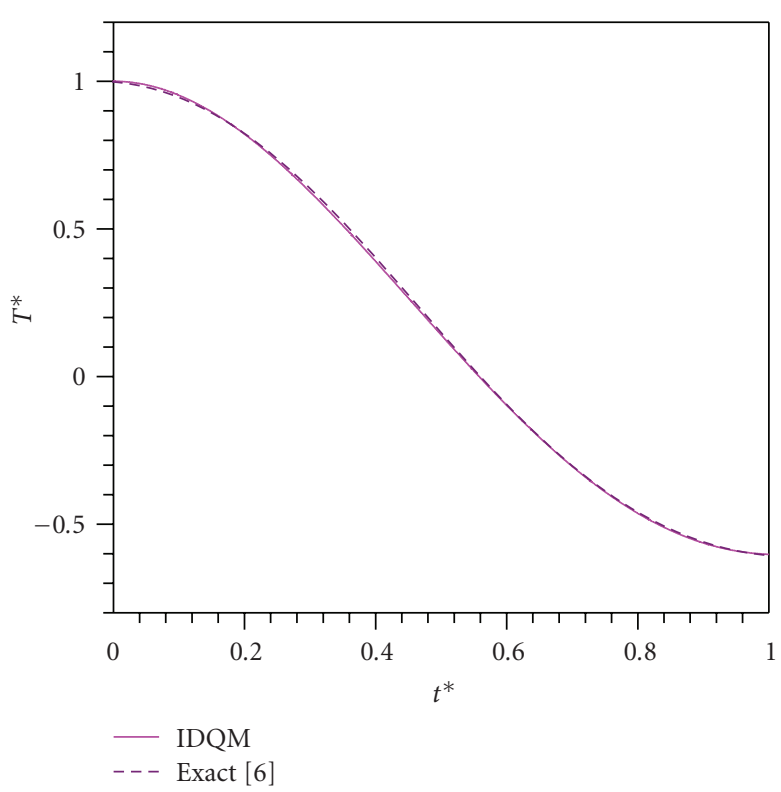

(b) Type (2)

Figure 1: The time history of the nondimensional temperature for both types of the initial conditions at $\xi=0.5$.

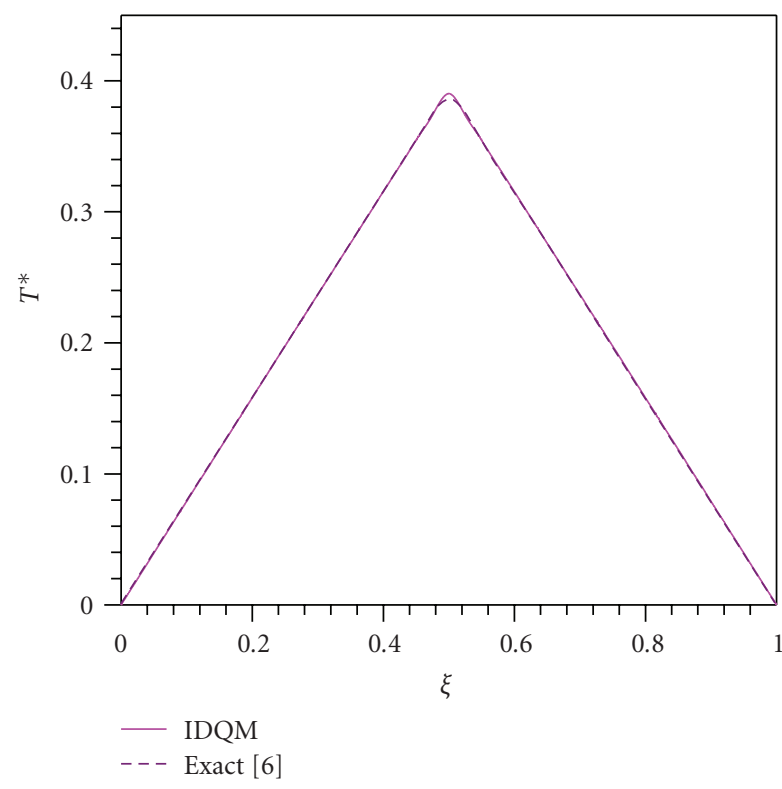

(a) Type (1)

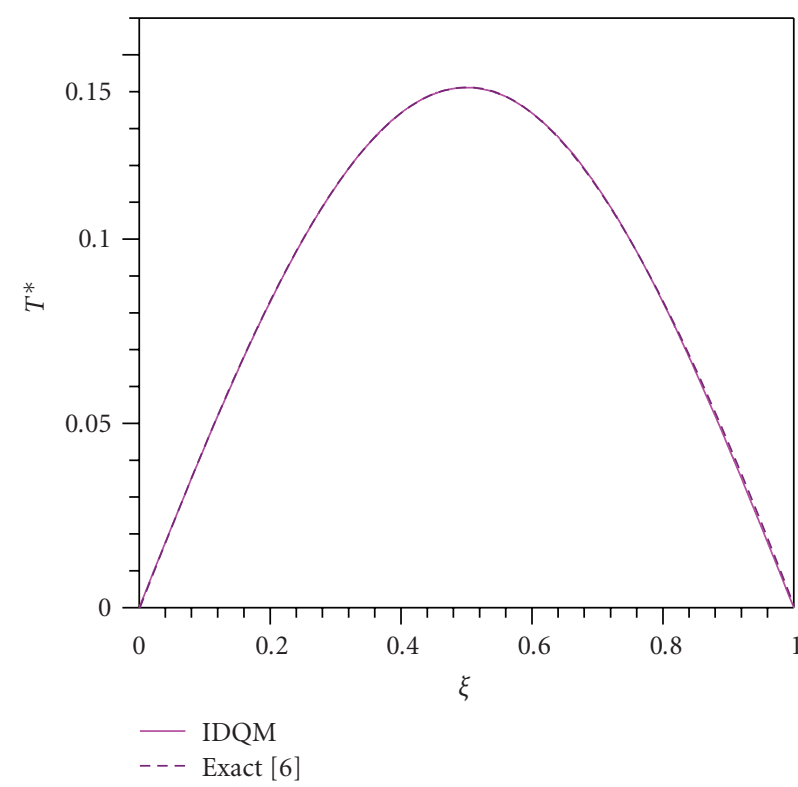

(b) Type (2)

FIgURE 2: The nondimensional temperature distribution for both types of the initial conditions along the $\xi$-axis at $t^{*}=0.5$.

temperature at one of its boundary investigated by Dorao [26] is performed. The boundary and initial conditions are taken as follows:

$$
\begin{array}{ll}
T(0, t)=\beta(t), & T\left(L_{x}, t\right)=0, \\
T(x, 0)=0, & \frac{\partial T(x, 0)}{\partial t}=0,
\end{array}
$$

where,

$$
\beta(t)= \begin{cases}\frac{1}{2}+\frac{3}{4}\left(\frac{2 t}{t_{0}}-1\right)-\frac{1}{4}\left(\frac{2 t}{t_{0}}-1\right)^{3} & \text { if } 0 \leq t \leq t_{0}, \\ 1 & \text { if } t \geq t_{0} .\end{cases}
$$

Also, the same parameters values as in Dorao [26] are adopted here; that is, the heat capacity $\gamma=1$, the relaxation 


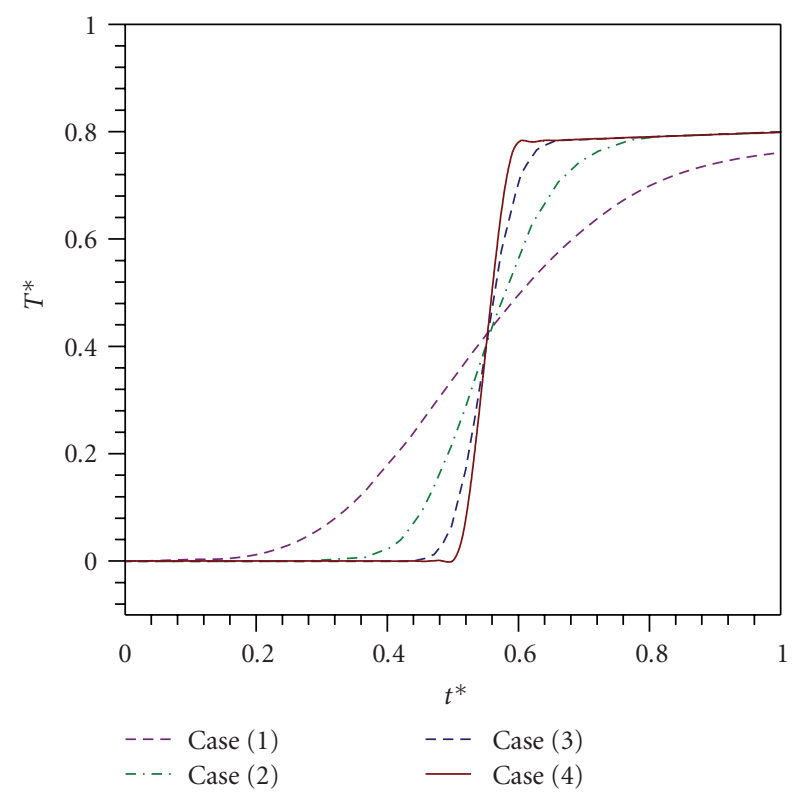

(a) The time history of the nondimensional temperature at $\xi=0.5$

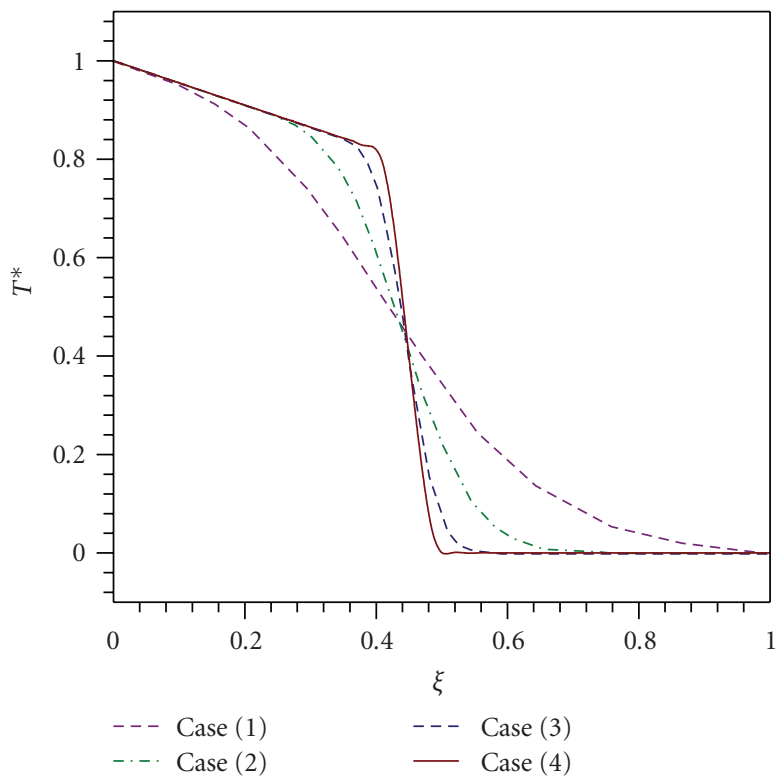

(b) Temperature along the $\xi$-axis for different methods at $t^{*}=0.5$

Figure 3: The convergence of the FEM. Case (1): FEM, (NDOF, CPU time (Second))=(169,0.3); Case (2): FEM, (5329,1); Case (3): FEM, $(56943,6)$; Case (4): IDQM, $(8591,1.4)$.

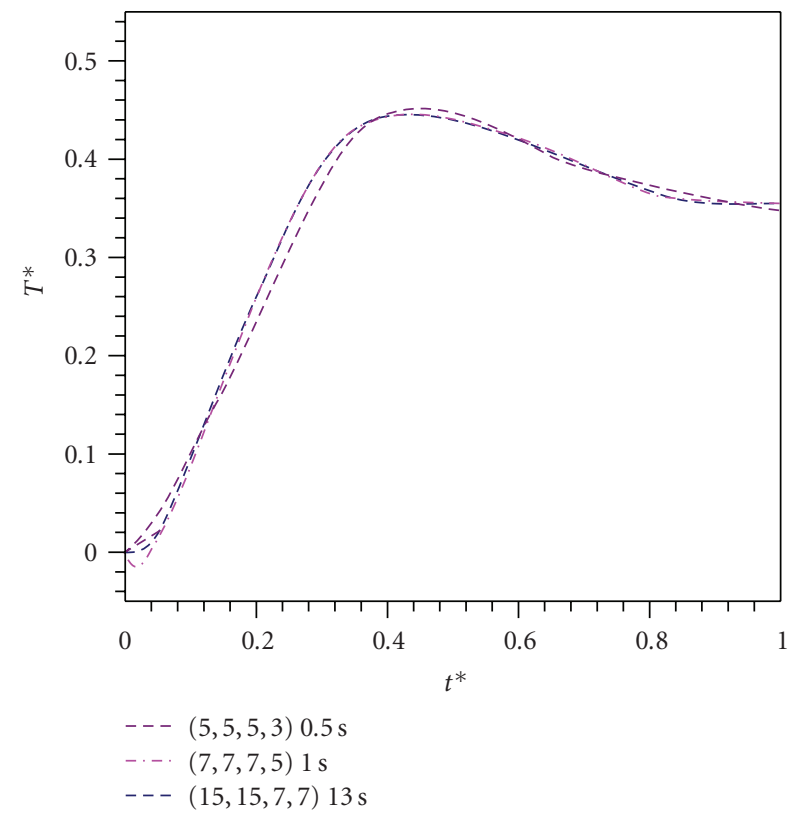

(a) The time history of the nondimensional temperature at $\eta=0.5$

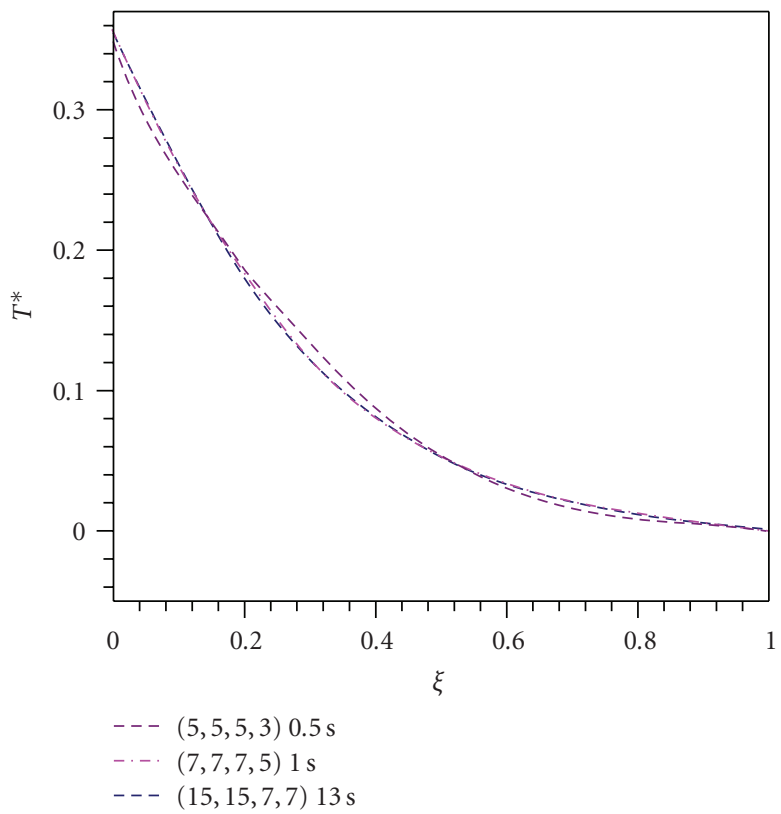

(b) The nondimensional temperature along the axis $\eta=0.5$ and $t^{*}=1$

FIGURE 4: The convergence behavior of IDQM against the number of DQ grid points.

time $\tau=1$, the thermal conductivity $k=1$, and $t_{0}=0.1$. By using the FEM, the linear element in conjunction with Galerkin scheme are employed to discretize the spatial and the temporal domain, respectively. The results of the both methods are compared in Figures 3(a) and 3(b) for the time history of the nondimensional temperature at $\xi=0.5$ and the nondimensional temperature along the $\zeta$-axis at $t^{*}=0.5$, respectively. It can be seen that by increasing the FEM number of degrees of freedom (NDOF), its results approaches to those of IDQM. It should also be noticed that the behaviors of the solutions are similar to those of Dorao [26].

3.2. Two-Dimensional Problems. In this section, the nonFourier thermal behaviors of a two-dimensional rectangular domain with temperature-dependent thermal conductivity 


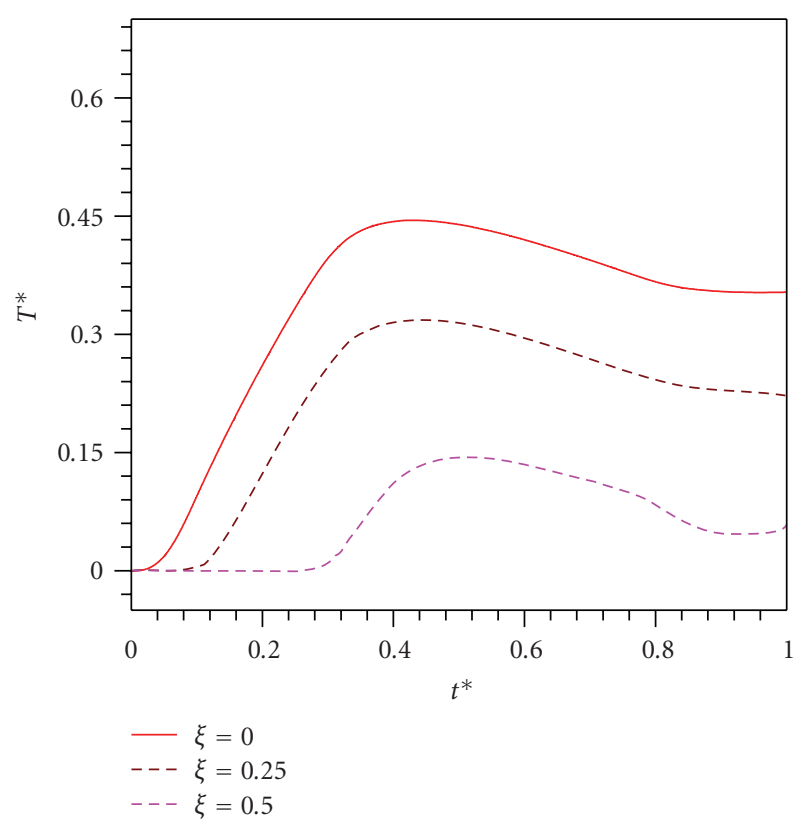

FIGURE 5: The time history of the nondimensional temperature at different points along the line $\eta=0.5$.

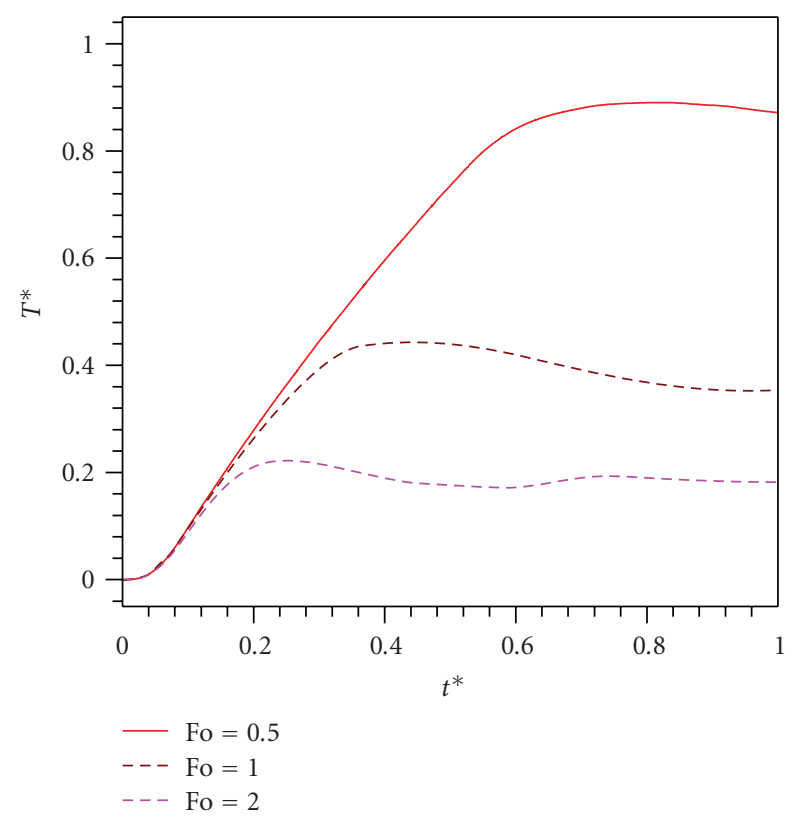

FIGURE 6: The time history of the nondimensional temperature at the point $(\xi=0, \eta=0.5)$ for different value of Fourier number.

are investigated using the IDQM. The domain are subjected to the following boundary and initial conditions:

$$
\begin{array}{rlrl}
-k \frac{\partial T(0, y, t)}{\partial x} & =q_{o} \beta(t), & T\left(L_{x}, y, t\right)=0, \\
T(x, 0, t) & =0, & T\left(x, L_{y}, t\right) & =0, \\
T(x, y, 0) & =0, & \frac{\partial T(x, y, 0)}{\partial t} & =0 .
\end{array}
$$

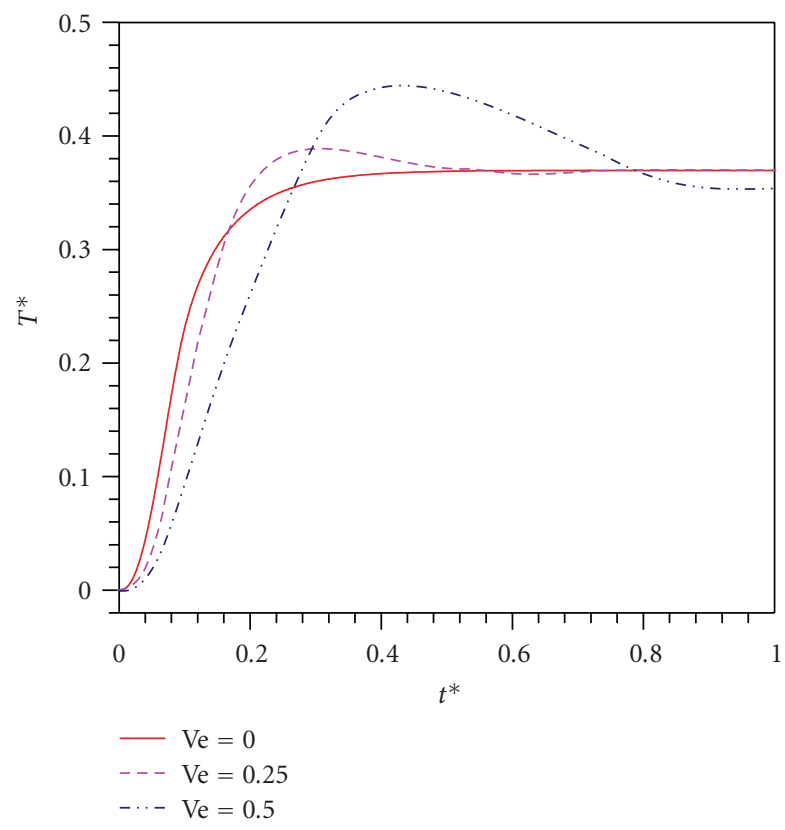

FIGURE 7: The effect of relaxation time on the time history of the nondimensional temperature at $(\xi=0, \eta=0.5)$.

Otherwise specified, the following values are used in the numerical computations:

$$
\text { Fo }=1, \quad \text { Ve }=0.5, \quad r=1, \quad T_{o}=1, \quad \lambda=0.1 .
$$

As a first example, the convergence of the method against the number of DQ grid points in the spatial and temporal domains $\left(N_{x}, N_{y}, N_{T}\right.$, and $\left.N_{I T}\right)$ is investigated. For this purpose, the time history of the nondimensional temperature at the point $(\xi=0, \eta=0.5)$ and also the nondimensional temperature along the axis $\eta=0.5$ and at the nondimensional time $t^{*}=1$ are presented in Figures 4(a) and 4(b), respectively. The fast rate of convergence of the method is quite evident.

After showing the convergence of the method, the parametric studies are performed.

The time histories of the nondimensional temperature at the different points on the centerline $(\eta=0.5)$ are shown in Figure 5 . The propagation of heat wave can be seen in this figure.

The time history of the nondimensional temperature at point $(\xi=0, \eta=0.5)$ and for the different values of Fourier number (Fo) is shown in Figure 6. It can be seen that as Fourier number or consistently total time increases, the nondimensional temperature approaches to its steady-state value.

The effect of relaxation time $\tau$, as appeared in Vernotte number $(\mathrm{Ve})$, on the time history of the nondimensional temperature of the point $(\xi=0, \eta=0.5)$ is shown in Figure 7. It is clear that by increasing the Vernotte number, the non-Fourier effect increases. It should be mentioned when $\mathrm{Ve}=0$, the results become equal to those obtained using Fourier law. 


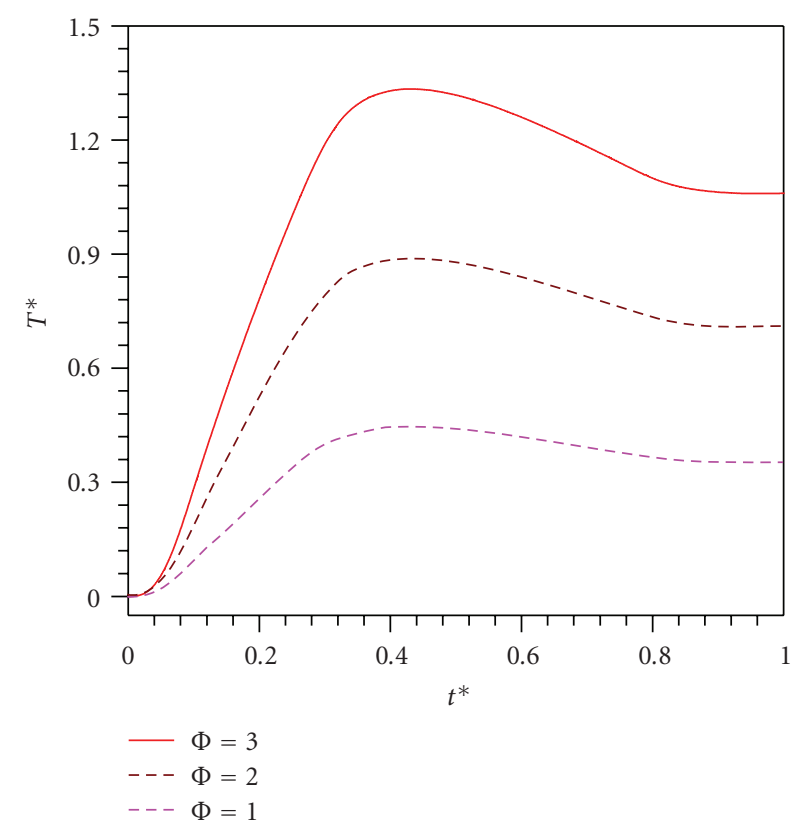

(a) The time history of the nondimensional temperature at $(\xi=0$, $\eta=0.5)$

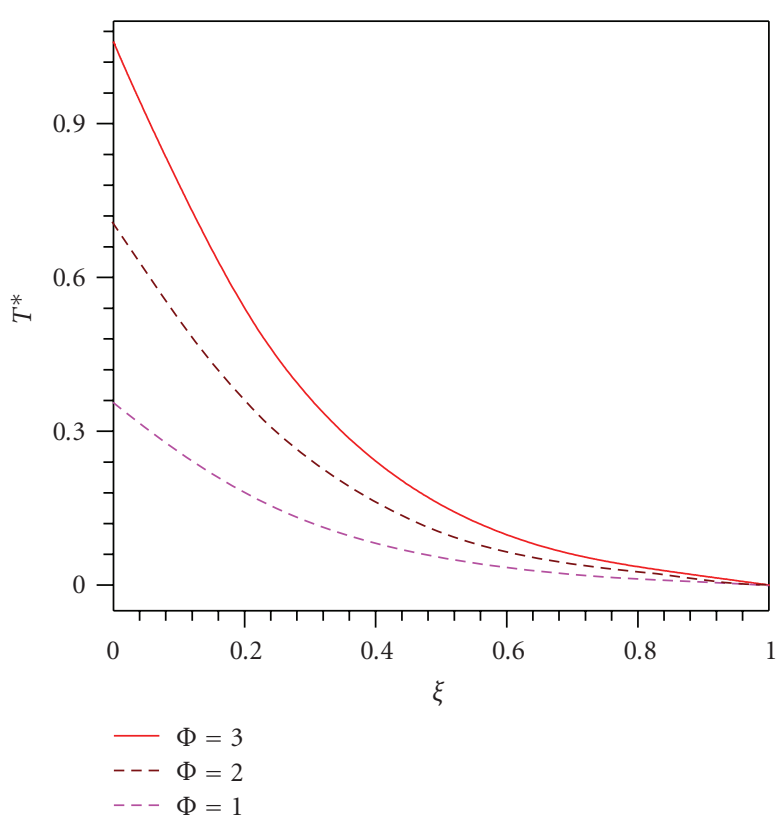

(b) $\eta=0.5$

FIgURE 8: The effects of heat supply at the boundary on the temperature distribution of the domain.

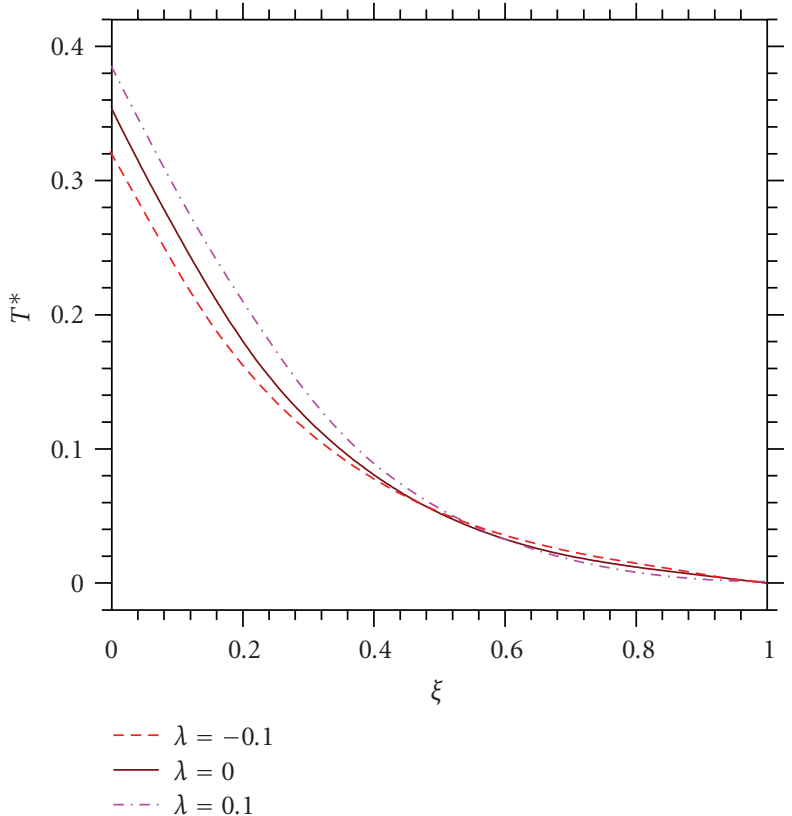

(a) $\eta=0.5$

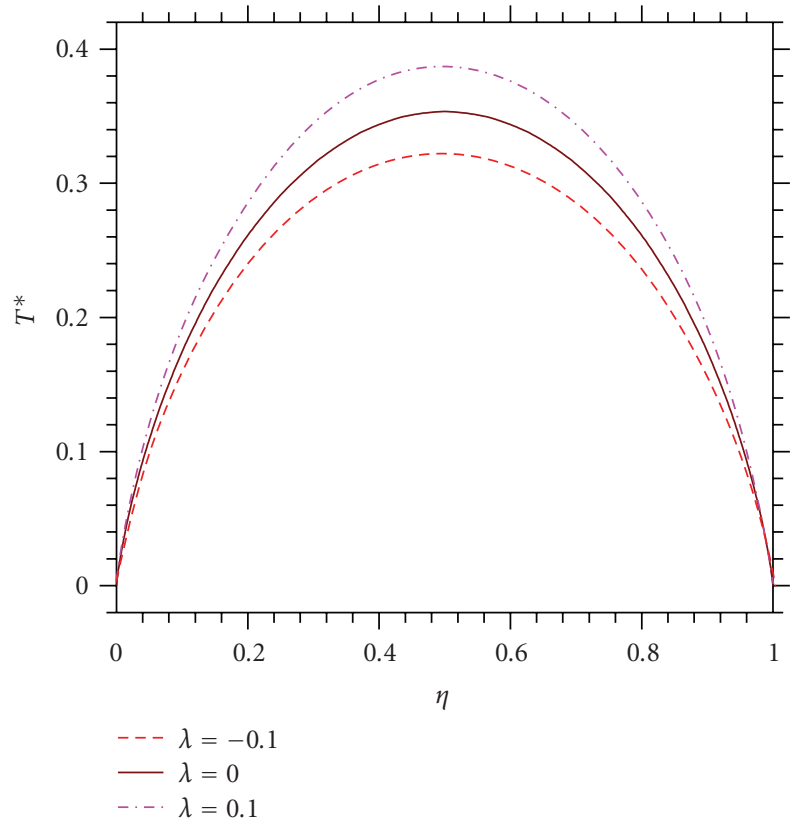

(b) $\xi=0$

FIGURE 9: The effect of temperature dependence of the thermal conductivity on the nondimensional temperature distribution along $\eta=0.5$ and $\xi=0$ axes.

Figures $8(\mathrm{a})$ and $8(\mathrm{~b})$ show the time history of the nondimensional temperature at point $(\zeta=0, \eta=0.5)$ and nondimensional temperature along the axis $\eta=0.5$ for different value of the nondimensional boundary heat flux $\Phi$, respectively. It can be seen that as the boundary heat flux increases, the temperature and temperature gradient increase.
The effect of temperature dependence of the thermal conductivity on the nondimensional temperature distribution of the domain is studied by showing the variation of the nondimensional temperature distribution along $\eta=0.5$ and $\zeta=0$ axes in Figures 9(a) and 9(b), respectively. The results are prepared for three different values of the thermal conductivity coefficient $\lambda$. Finally, the effect of the aspect 


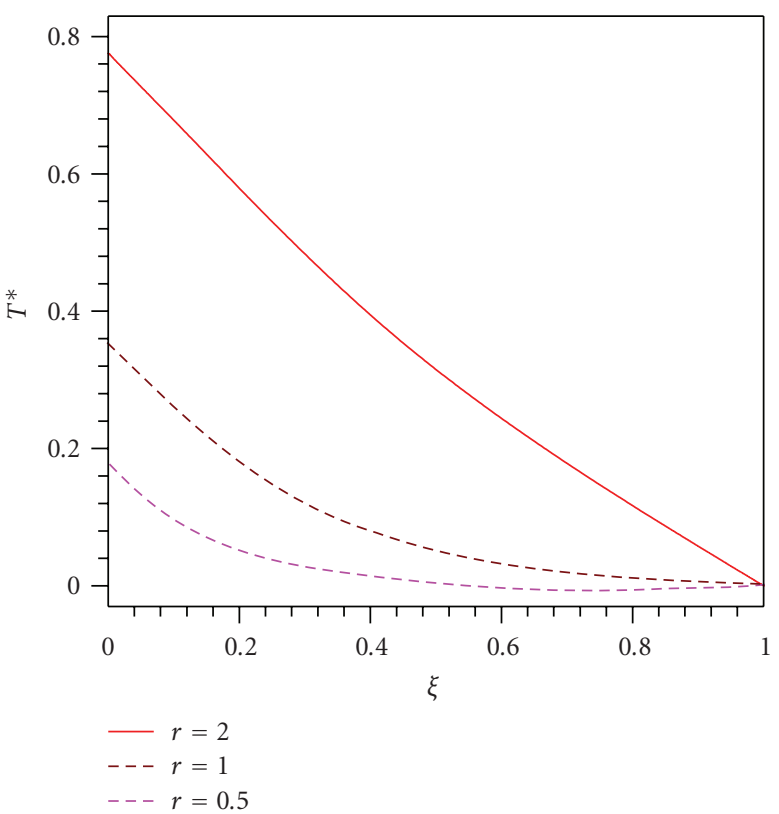

(a) $\eta=0.5$

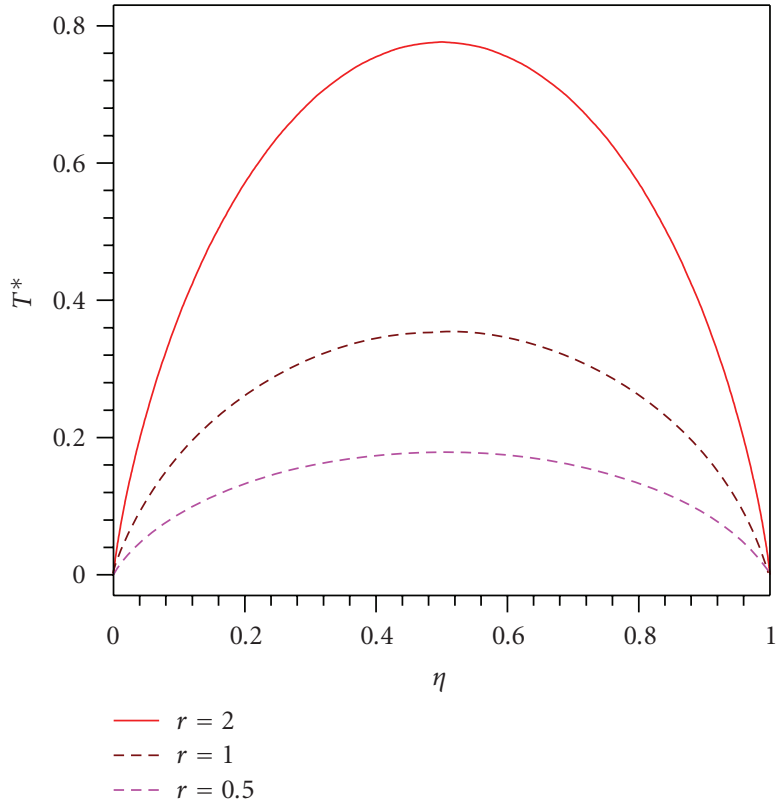

(b) $\xi=0$

FIgURE 10: The influence of the aspect ratio $(r)$ on the temperature distribution of the domain.

ratio $(r)$ on the temperature distribution in the domain is shown in Figure 10. One can see that by increasing the aspect ratio, the temperature at the domain grid point increases.

\section{Conclusions}

As a first attempt, the differential quadrature method (DQM) is employed to study the one- and two-dimensional heat wave propagation in a medium with temperature-dependent thermal conductivity. Both the spatial as well as the temporal domain is discretized using the DQM. The superior accuracy with fewer degrees of freedom than conventional finite element method (FEM) of DQM is shown. The convergence and accuracy of the method is demonstrated. The effects of different parameters such as relaxation time, Fourier number, temperature dependency of thermal conductivity and aspect ratio of the domain on the temperature distribution of the medium are studied. It is shown that these parameters have significant effect on the temperature distribution of the domain.

\section{Nomenclature}

$A^{\zeta}$ : The normalized weighting coefficients of the first-order derivative $(\zeta=x, y, t)$

$B^{\zeta}$ : The normalized weighting coefficients of the second-order derivative $(\zeta=x, y, t)$

$C$ : Thermal wave speed

$C_{p}$ : Heat capacity

Fo: Fourier number $\left(=\alpha_{o} t_{\text {total }} / L_{x}^{2}\right)$

$g$ : Internal heat generation source $k$ : Thermal conductivity

$k_{o}$ : Thermal conductivity at reference temperature

$L_{x}$ : Length of the domain along the $x$-direction

$L_{y}$ : Length of the domain along the $y$-direction

$N_{I T}$ : Number of temporal subdomain

$N_{T}$ : Number of grid points in of a temporal subdomain

$N_{x}$ : Number of grid points in the $x$-direction

$N_{y}$ : Number of grid points in the $y$-direction

$q$ : Heat flux

$t$ : Time

$t_{\text {total }}:$ Total time

$t^{*}:$ Nondimensional time

T: Temperature

$T_{o}$ : Reference temperature

$T^{*}$ : Nondimensional temperature $\left(=T / T_{o}\right)$

$V e$ : Vernotte number $\left(=\sqrt{\alpha_{o} \tau} / L_{x}\right)$.

\section{Greek Symbols}

$\alpha$ : Thermal diffusivity $\left(k / \rho C_{p}\right)$

$\alpha_{o}$ : Thermal diffusivity at reference temperature

$\Phi$ : Nondimensional boundary heat flux $\left(=q_{o} L_{x} / k_{o} T_{o}\right)$

$\eta$ : Nondimensional coordinate variable along the $y$-axis $\left(=y / L_{y}\right)$

$\lambda$ : Thermal conductivity coefficient

$\rho$ : Density

$\tau:$ Relaxation time $\left(=\alpha / C^{2}\right)$

$\xi$ : Nondimensional coordinate variable along the $x$-axis $\left(=x / L_{x}\right)$. 


\section{References}

[1] T. Sanderson, C. Ume, and J. Jarzynski, "Hyperbolic heat conduction effects caused by temporally modulated laser pulses," Ultrasonics, vol. 33, no. 6, pp. 423-427, 1995.

[2] J. Y. Lin, "The non-Fourier effect on the fin performance under periodic thermal conditions," Applied Mathematical Modelling, vol. 22, no. 8, pp. 629-640, 1998.

[3] P. J. Antaki, "Importance of nonFourier heat conduction in solid-phase reactions," Combustion and Flame, vol. 112, no. 3, pp. 329-341, 1998.

[4] W. B. Lor and H. S. Chu, "Effect of interface thermal resistance on heat transfer in a composite medium using the thermal wave model," International Journal of Heat and Mass Transfer, vol. 43, no. 5, pp. 653-663, 2000.

[5] L. H. Liu, H. P. Tan, and T. W. Tong, "Non-Fourier effects on transient temperature response in semitransparent medium caused by laser pulse," International Journal of Heat and Mass Transfer, vol. 44, no. 17, pp. 3335-3344, 2001.

[6] L. Wang, "Solution structure of hyperbolic heat-conduction equation," International Journal of Heat and Mass Transfer, vol. 43, no. 3, pp. 365-373, 2000.

[7] R. Cai, C. Gou, and H. Li, "Algebraically explicit analytical solutions of unsteady 3-D nonlinear non-Fourier (hyperbolic) heat conduction," International Journal of Thermal Sciences, vol. 45, no. 9, pp. 893-896, 2006.

[8] A. Moosaie, "Non-Fourier heat conduction in a finite medium subjected to arbitrary periodic surface disturbance," International Communications in Heat and Mass Transfer, vol. 34, no. 8, pp. 996-1002, 2007.

[9] J. S. Loh, I. A. Azid, K. N. Seetharamu, and G. A. Quadir, "Fast transient thermal analysis of Fourier and non-Fourier heat conduction," International Journal of Heat and Mass Transfer, vol. 50, no. 21-22, pp. 4400-4408, 2007.

[10] R. Shirmohammadi and A. Moosaie, "Non-Fourier heat conduction in a hollow sphere with periodic surface heat flux," International Communications in Heat and Mass Transfer, vol. 36, no. 8, pp. 827-833, 2009.

[11] M. Jadidi, "Non-fourier heat conduction in a long cylindrical media with insulated boundaries and arbitrary initial conditions," Australian Journal of Basic and Applied Sciences, vol. 3, no. 2, pp. 652-663, 2009.

[12] G. F. Carey and M. Tsai, "Hyperbolic heat transfer with reflection," Numerical Heat Transfer Part A, vol. 5, no. 3, pp. 309-327, 1982.

[13] D. E. Glass, M. N. Oezisik, D. S. McRae, and B. Vick, "The numerical solution of hyperbolic heat conduction," Numerical Heat Transfer Part A, vol. 8, no. 4, pp. 497-504, 1985.

[14] K. K. Tamma and S. B. Railkar, "Specially tailored transfiniteelement formulations for hyperbolic heat conduction involving non-Fourier effects," Numerical Heat Transfer, Part B, vol. 15, no. 2, pp. 211-226, 1989.

[15] C. Han-Taw and L. Jae-Yuh, "Numerical analysis for hyperbolic heat conduction," International Journal of Heat and Mass Transfer, vol. 36, no. 11, pp. 2891-2898, 1993.

[16] C. Han-Taw and L. Jae-Yuh, "Analysis of two-dimensional hyperbolic heat conduction problems," International Journal of Heat and Mass Transfer, vol. 37, no. 1, pp. 153-164, 1994.

[17] W. K. Yeung and T. T. Lam, "A numerical scheme for nonfourier heat conduction, part I: one-dimensional problem formulation and applications," Numerical Heat Transfer, Part B, vol. 33, no. 2, pp. 215-233, 1998.

[18] P. T. Hsu and Y. H. Chu, "An inverse non-Fourier heat conduction problem approach for estimating the boundary condition in electronic device," Applied Mathematical Modelling, vol. 28, no. 7, pp. 639-652, 2004.

[19] K. C. Liu and H. T. Chen, "Numerical analysis for the hyperbolic heat conduction problem under a pulsed surface disturbance," Applied Mathematics and Computation, vol. 159, no. 3, pp. 887-901, 2004.

[20] W. Wu and X. Li, "Application of the time discontinuous Galerkin finite element method to heat wave simulation," International Journal of Heat and Mass Transfer, vol. 49, no. 9-10, pp. 1679-1684, 2006.

[21] X. Li, D. Yao, and R. W. Lewis, "A discontinuous Galerkin finite element method for dynamic and wave propagation problems in non-linear solids and saturated porous media," International Journal for Numerical Methods in Engineering, vol. 57, no. 12, pp. 1775-1800, 2003.

[22] W. Wu and X. Li, "Application of the time discontinuous Galerkin finite element method to heat wave simulation," International Journal of Heat and Mass Transfer, vol. 49, no. 9-10, pp. 1679-1684, 2006.

[23] T. M. Chen, "Numerical solution of hyperbolic heat conduction in thin surface layers," International Journal of Heat and Mass Transfer, vol. 50, no. 21-22, pp. 4424-4429, 2007.

[24] A. P. Reverberi, P. Bagnerini, L. Maga, and A. G. Bruzzone, "On the non-linear Maxwell-Cattaneo equation with nonconstant diffusivity: shock and discontinuity waves," International Journal of Heat and Mass Transfer, vol. 51, no. 21-22, pp. 5327-5332, 2008.

[25] C. Y. Yang, "Direct and inverse solutions of the twodimensional hyperbolic heat conduction problems," Applied Mathematical Modelling, vol. 33, no. 6, pp. 2907-2918, 2009.

[26] C. A. Dorao, "Simulation of thermal disturbances with finite wave speeds using a high order method," Journal of Computational and Applied Mathematics, vol. 231, no. 2, pp. 637-647, 2009.

[27] T. M. Chen, "A hybrid Green's function method for the hyperbolic heat conduction problems," International Journal of Heat and Mass Transfer, vol. 52, no. 19-20, pp. 4273-4278, 2009.

[28] T. M. Chen and C. C. Chen, "Numerical solution for the hyperbolic heat conduction problems in the radial-spherical coordinate system using a hybrid Green's function method," International Journal of Thermal Sciences, 2010.

[29] C. C. Wang, "Direct and inverse solutions with non-Fourier effect on the irregular shape," International Journal of Heat and Mass Transfer, vol. 53, no. 13-14, pp. 2685-2693, 2010.

[30] S. Andarwa and H. Basirat Tabrizi, "Non-Fourier effect in the presence of coupled heat and moisture transfer," International Journal of Heat and Mass Transfer, vol. 53, no. 15-16, pp. 30803087, 2010.

[31] M. H. Hsu, "Differential quadrature method for solving hyperbolic heat conduction problems," Tamkang Journal of Science and Engineering, vol. 12, no. 3, pp. 331-338, 2009.

[32] P. Malekzadeh and H. Rahideh, "IDQ two-dimensional nonlinear transient heat transfer analysis of variable section annular fins," Energy Conversion and Management, vol. 48, no. 1, pp. 269-276, 2007.

[33] P. Malekzadeh, H. Rahideh, and G. Karami, "A differential quadrature element method for nonlinear transient heat transfer analysis of extended surfaces," Numerical Heat Transfer; Part A, vol. 49, no. 5, pp. 511-523, 2006.

[34] M. R. G. Haghighi, M. Eghtesad, and P. Malekzadeh, "A coupled differential quadrature and finite element method for 3-D transient heat transfer analysis of functionally graded 
thick plates," Numerical Heat Transfer, Part B, vol. 53, no. 4, pp. 358-373, 2008.

[35] M. R. G. Haghighi, M. Eghtesad, P. Malekzadeh, and D. S. Necsulescu, "Two-dimensional inverse heat transfer analysis of functionally graded materials in estimating time-dependent surface heat flux," Numerical Heat Transfer; Part A, vol. 54, no. 7, pp. 744-762, 2008.

[36] P. Malekzadeh, A. R. Fiouz, and H. Razi, "Three-dimensional dynamic analysis of laminated composite plates subjected to moving load," Composite Structures, vol. 90, no. 2, pp. 105$114,2009$. 

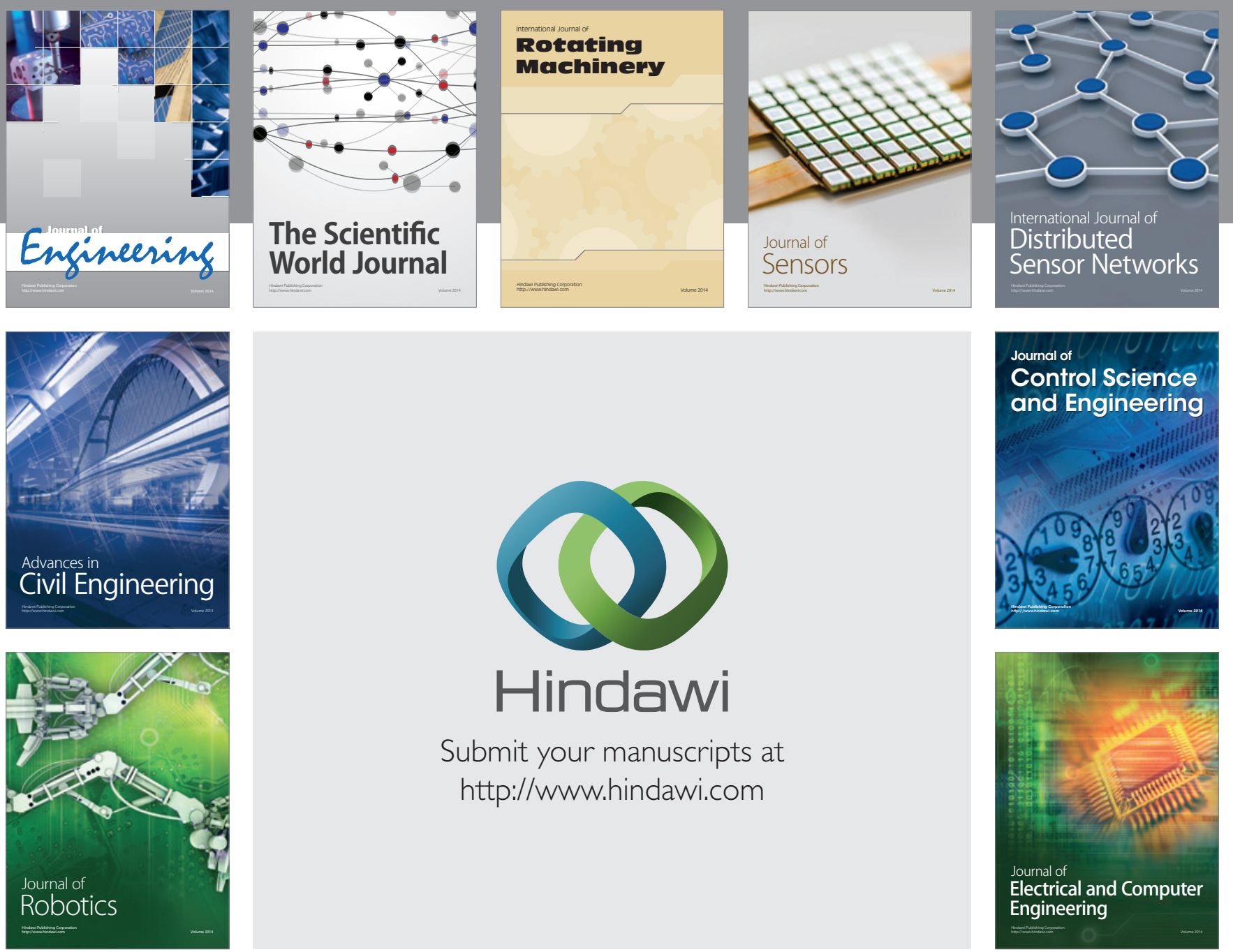

Submit your manuscripts at

http://www.hindawi.com
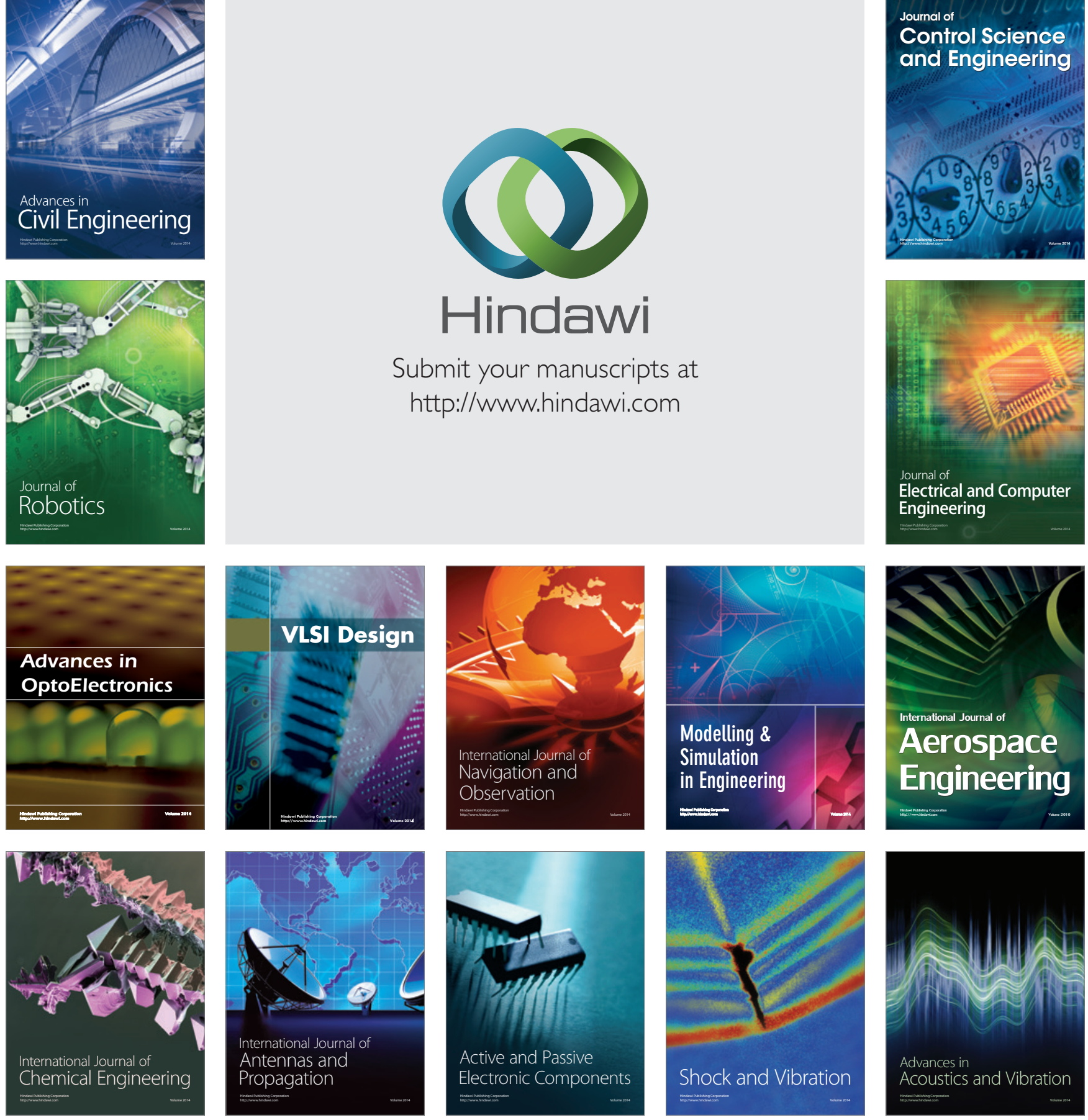